\title{
DESIGN OF A SIX-AXIS MESO-SCALE NANOPOSITIONER DRIVEN BY MOVING- COIL MICROACTUATORS
}

Dariusz S. Golda and Martin L. Culpepper

Massachusetts Institute of Technology, Cambridge, Massachusetts, USA

\begin{abstract}
This paper presents the design, fabrication, and characterization of a high-speed, six-axis, meso-scale nanopositioner with applications in nanomanufacturing, probebased microscopy, and data storage. The nanopositioner consists of a silicon flexure bearing, sample stage, and magnetic movingcoil microactuators. A new fabrication process was generated to integrate a silicon flexure and planar copper coils. The nanopositioner was measured to have a range of 10 micrometers and 1 micrometer in the lateral and out-of-plane directions, respectively, an angular range of 0.5 degrees, and a first mode resonant frequency at $900 \mathrm{~Hz}$. Open-loop calibration has been shown to minimize parasitic in-plane motion to less than $100 \mathrm{~nm}$.
\end{abstract}

\section{INTRODUCTION}

Nanopositioners enable the motion control of large or small parts with nanometer-level or better precision in multiple axes. They therefore set limits on the ability to measure and manipulate physical systems. Macro-scale nanopositioners are designed to position payloads such as wafers and optical elements. The large size (tens of centimeters) and mass (kilograms) of macro-scale nanopositioners limits their bandwidth to a few hundred Hertz. They are inherently sensitive to thermal drift in position and orientation because of their large size. Meso- or millimeter-scale nanopositioners enable a combination of larger bandwidth, improved thermal stability, portability, and the potential for parallel operation in a small package. Meso-scale nanopositioners are suited to position modest payloads such as probe tips, millimeter-sized data storage media, and cell samples. Meso-scale nanopositioners are envisioned to impact applications in data storage [1], nanomanufacturing [2], and probe-based metrology. These applications may benefit from low-cost, portable, multi-axis nanopositioners that position samples with (i) nanometer-level precision, (ii) at bandwidths of $100 \mathrm{~s} \mathrm{~Hz}$ and (iii) over a working envelope that is larger than $10 \times 10 \times 10 \mu \mathrm{m}^{3}$. Previously reported meso-scale nanopositioners were limited to five axes of motion or less, and maximum force density has limited the range or natural frequency when using flexure bearings [3-5]. An electrothermal six-axis meso-scale system has been demonstrated [6]; however its range was limited to a few micrometers.

The paper presents the design, fabrication, and experimental characterization of a meso-scale, six-axis nanopositioner wherein silicon flexure bearings and moving-coil microactuators have been combined to yield improved performance over previous designs.

\section{NANOPOSITIONER DESIGN System Concept}

The six-axis nanopositioner is designed to (1) operate in open-loop with a range-of-motion that is larger than 10 micrometers in the X-, Y- and Z- directions; and 0.5 degrees in the $\theta_{\mathrm{x}}, \theta_{\mathrm{y}}$ and $\theta_{\mathrm{z}}$ directions, (2) possess a natural frequency of several $100 \mathrm{~Hz}$, (3) exhibit better than $10 \mathrm{~nm}$ resolution and $50 \mathrm{~nm}$ repeatability in open-loop, and (4) possess a footprint of less than $20 \mathrm{~mm}$ on a side. The nanopositioning system that was constructed in this work is shown in Figure 1. The system is comprised of three sets of magnet-coil microactuators, a silicon flexure mechanism, sample stage, alignment features, and magnet fixture package. The microfabricated silicon chip was aligned to the permanent magnets with DRIE alignment features that mate to pins in a precision-machined magnet alignment fixture.

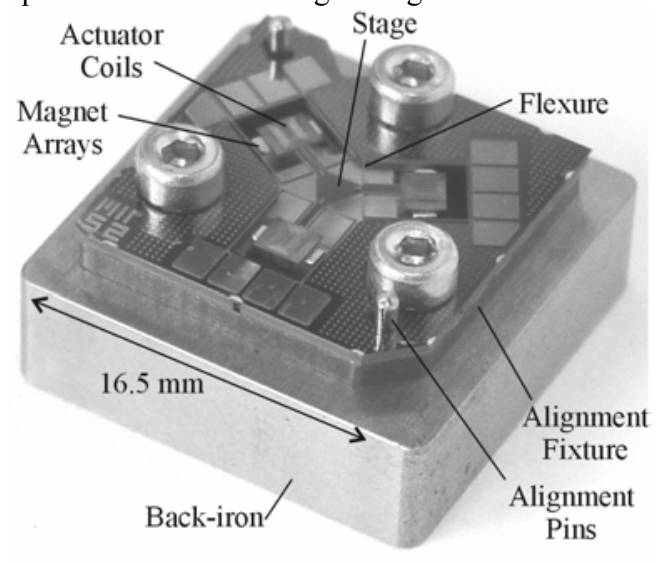

Figure 1: Six-axis nanopositioner with magnet fixture.

The six-axis actuation concept is based upon the HexFlex flexure system that is shown in Figure 2 [7]. Two orthogonal forces were applied to the planar flexure bearing at each of the three actuator paddles in the lateral and out-of-plane directions. Six independent actuator forces were combined through linear bending and torsion of the flexure bearings to yield six-axis motion of the stage in the X-, Y-, Z-, $\theta_{\mathrm{x}^{-}}, \theta_{\mathrm{y}^{-}}$and $\theta_{\mathrm{z}^{-}}$directions.

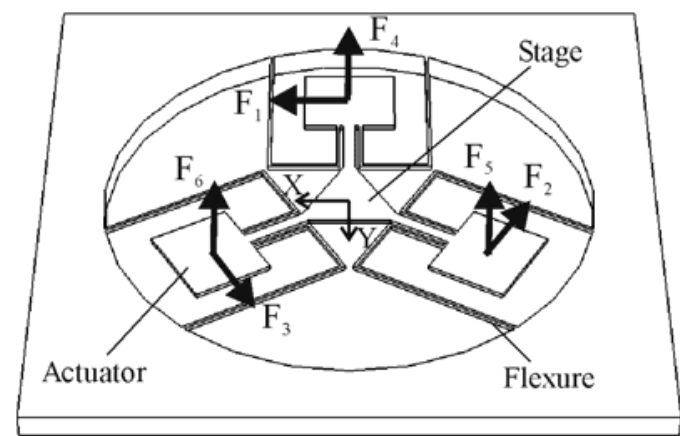

Figure 2: Meso-scale HexFlex six-axis actuation concept.

The actuator inputs, $\mathbf{i}$, that are required to generate a commanded stage displacement, $\mathbf{x}$, is given by:

$$
\mathbf{i}=\left(T K_{a}\right)^{-1} K \mathbf{x}
$$

where $T$ is the force transformation matrix, $K_{a}$ is the actuation matrix, and $K$ is the stiffness matrix of the flexure bearing. The actuation matrix relates the input currents to the actuator forces. The force transformation matrix transforms the forces applied at the paddles to a set of forces applied to the stage. The nanopositioner requires three actuators that are capable of generating orthogonal and independent linear forces in two axes in order to achieve six-axis motion in the system. 


\section{Actuator Concept}

A new two-axis, magnetic moving-coil actuator was created to drive the nanopositioner [8]. Each actuator consists of stacked micro-coils that are suspended above an alternating array of $1 \mathrm{~mm}^{3}$ permanent magnets via a silicon flexure system as shown in Figure 3. The silicon flexure beams support copper traces that provide electrical current to each of the actuator coils.

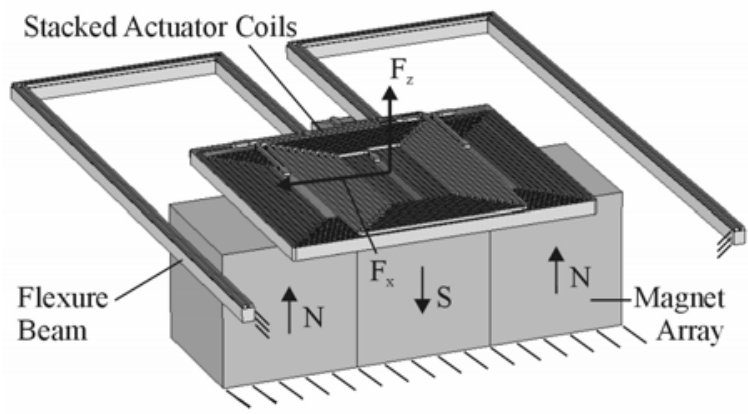

Figure 3: Rendering of two-axis moving-coil actuator concept.

The actuator consists of two independent coils that are stacked upon each other and that apply in-plane and out-of-plane forces to the flexure as shown in Figure 4.

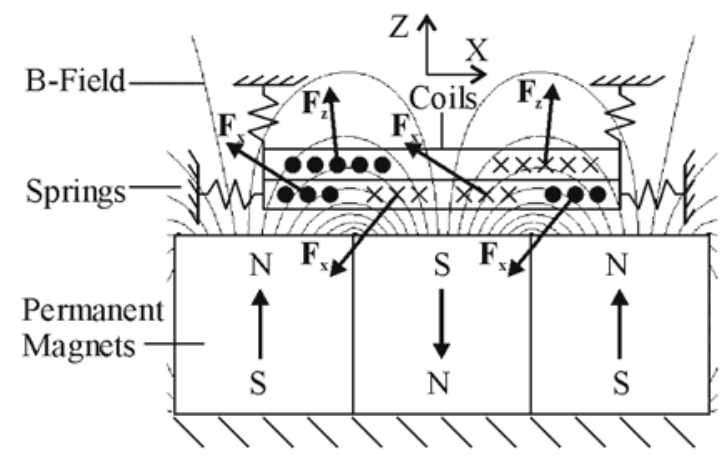

Figure 4: Cross-section of the actuator concept that illustrates the directions of Lorentz forces that act on the y-directed coil segments

The working coil segments that are directed in the Y-direction interact with the permanent magnet field to generate Lorentz forces in the X- and Z-directions. An upper "racetrack" coil generates a net Z-directed force, while a lower "figure-eight" coil generates a net X-directed force. The force properties of the actuator were optimized by adjusting the lateral dimensions of the coils [8]. The actuator inputs are combined to control the stage position in six axes. This actuator outperforms previous designs as it exerts orthogonal, linear forces while minimizing parasitic forces.

\section{FABRICATION}

A new microfabrication process was created to integrate multi-layer, copper actuator coils, silicon flexure bearings, and alignment features. Figure 5 shows the process flow diagram. The process was developed at the MIT Microsystems Technology Lab (MTL). The process used SOI wafers with a $100 \mu \mathrm{m}$ device layer and $1 \mu \mathrm{m}$ buried oxide. Trenches for the copper coil mold with cross-sections of $30 \mu \mathrm{m} \times 30 \mu \mathrm{m}$ were created via DRIE in step (2) and then thermally oxidized in step (3). A Ti/Cu seed layer was sputtered in step (4). The trenches were electroplated into a silicon mold using a deep-filling chemistry at Nexx Systems (Billerica, MA) in step (5). The overflow copper was polished in step (6) by chemical-mechanical planarization (CMP). In step (7) a PECVD silicon dioxide interlayer dielectric was deposited and patterned by wet etch to electrically isolate the copper coils. The oxide pattern was also used as the etch mask for the flexure beams in step (12). A second $\mathrm{Ti} / \mathrm{Cu}$ seed layer was evaporated in step (8). The second copper coil layer was then electroplated into a photoresist mold in step (9). The resist-molded coils had a cross-section width and height of $25 \mu \mathrm{m} \times 28 \mu \mathrm{m}$. The resist was removed with acetone after plating and the $\mathrm{Ti} / \mathrm{Cu}$ seed layer was wet-etched. The backside of the wafer was etched with DRIE to the buried oxide in step (10) in order to define the thickness of the device. Photoresist was used as the etch mask. The buried oxide was then etched with vapor HF in step (11). The front of the wafer was protected with photoresist in order to prevent the HF vapors from attacking the PECVD oxide and the Ti adhesion layer beneath the exposed coils. The upper copper coil layer and silicon dioxide were then used as a mask to etch the silicon flexure mechanism and alignment features with DRIE in step (12). The wafers were mounted on a bare silicon wafer in this step in order to enable the through-etch. The exposed copper structures were not observed to be damaged by the DRIE etch gases. The mean width of the silicon flexure beams after etching was less than $80 \mu \mathrm{m}$. The wafer was dismounted in acetone and cleaned in low-power oxygen plasma after etching of the flexures and alignment features. The dies were then separated by laser ablation in step (13). Fabrication process details are available in Ref. [9].

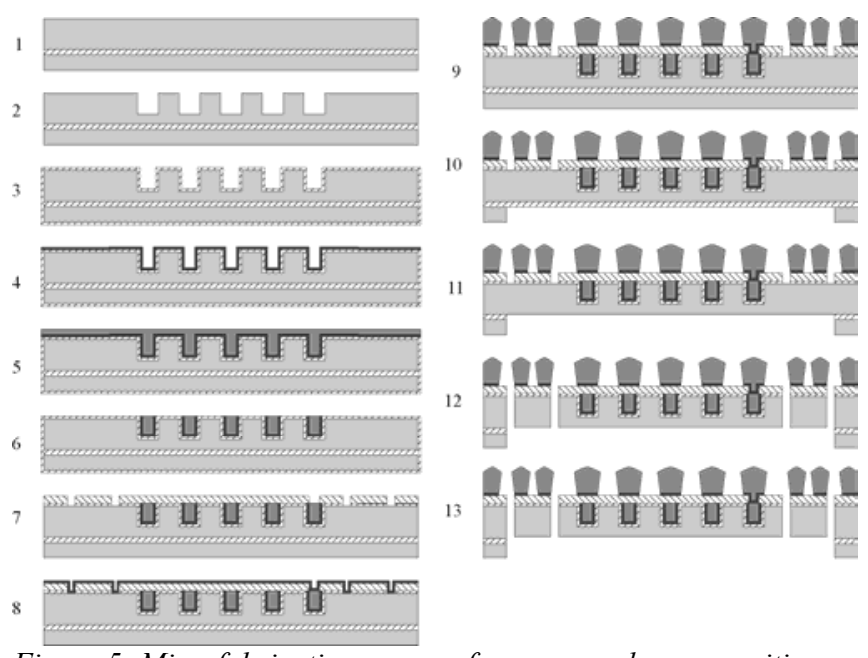

Figure 5: Microfabrication process for meso-scale nanopositioner

Figure 6 shows an optical image of the completed chip. The chip contains copper actuator coils and leads, silicon flexures, alignment features, fastener holes, and bond pads.

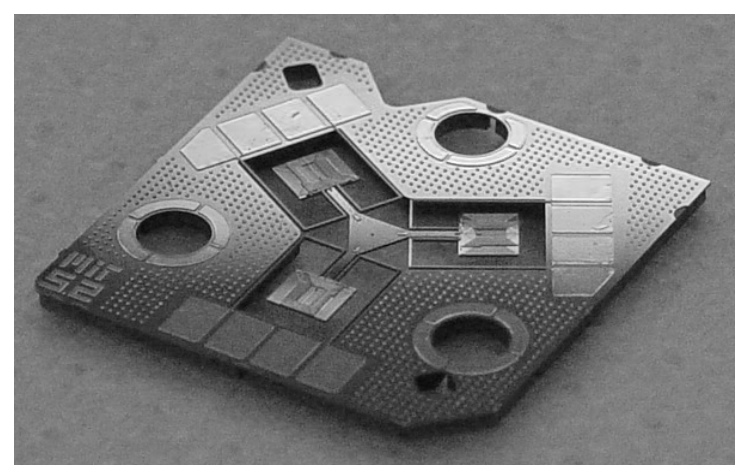

Figure 6: Optical images of the completed nanopositioner die. 
The chip was then aligned and fastened to a fixture that contained permanent magnet arrays, alignment features, and a permeable back-iron as shown in Figure 1.

Figure 7 shows the released actuator paddle after assembly to the permanent magnet fixture. The coils, jumpers, and via plugs are visible on the paddle. Permanent magnets are visible beneath the actuator paddle. The air gap between the paddle and magnets was set by the thickness of the alignment fixture and adjusted with polyester shims. Debris that accumulated below the magnets, and assembly errors, resulted in variations in the position of magnets with respect to the coils. The mean, minimum, and maximum air gaps were measured to be 80,20 , and $120 \mu \mathrm{m}$, respectively.

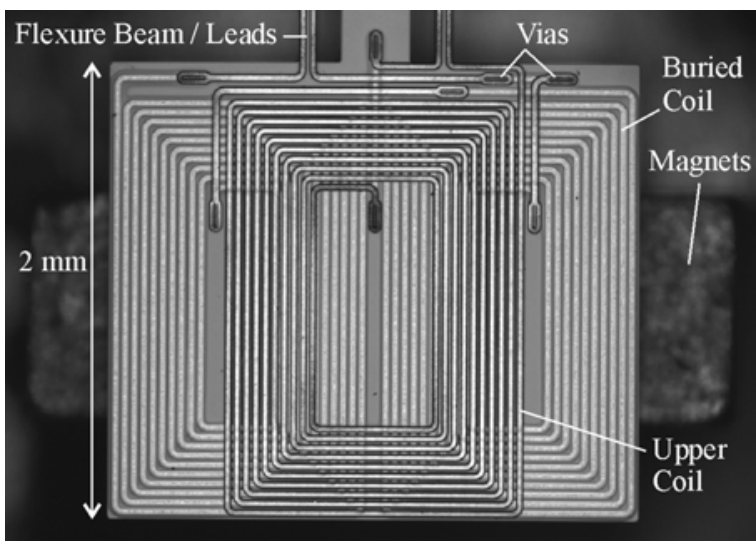

Figure 7: Optical image of a released actuator paddle that is suspended above a magnet array.

\section{EXPERIMENTAL RESULTS}

In-plane frequency response and static range-of-motion were measured at the stage center with a uMech MEMS Motion Analyzer. The out-of-plane frequency response and step response were measured at the stage center with a Polytec MSA-400 laser vibrometer. The device was driven with a 6 channel current controller that possessed an electrical bandwidth of over $3 \mathrm{MHz}$.

\section{Frequency Response}

Figure 8 shows the measured in-plane frequency response of the nanopositioner. In this experiment, the system was driven by three in-plane actuators. The first mode frequency was observed to be an underdamped rotational mode about the Z-axis at $900 \mathrm{~Hz}$. The peak at $1780 \mathrm{~Hz}$ represents the $\mathrm{X}$ - and $\mathrm{Y}$ - translation modes.
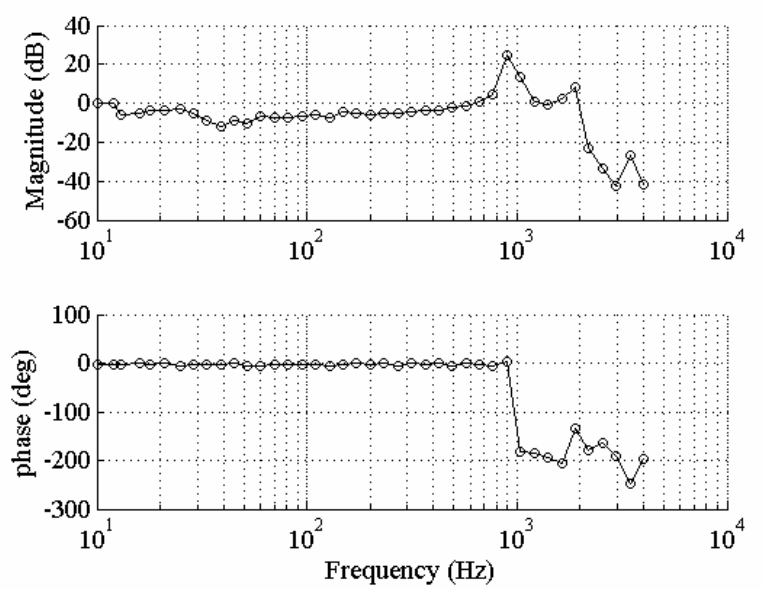

Figure 8: Measured frequency response for pre-calibrated actuation in the $\theta_{z}$-direction.
Figure 9 shows the measured out-of-plane frequency response due to one out-of-plane actuator input. The underdamped peak near $1 \mathrm{kHz}$ is composed of two closely spaced resonances. The lowest resonant frequency was measured at $1040 \mathrm{~Hz}$ and observed to be a tilting mode about the $\mathrm{X}$-axis. The translational mode in the Z-direction was measured at $1060 \mathrm{~Hz}$. The higher order modes at $4.5 \mathrm{kHz}$ and $10 \mathrm{kHz}$ are structural deformation modes of the stage.

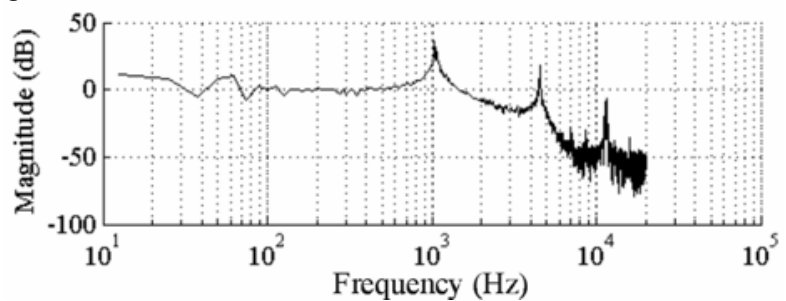

Figure 9: Measured frequency response for pre-calibrated actuation in the Z-direction.

\section{In-Plane Static Response}

The nanopositioner was calibrated for open loop operation in the plane by constructing a $3 \times 3$ calibration matrix from experimental data. The resultant $\mathrm{X}-, \mathrm{Y}-$, and $\theta_{\mathrm{z}}$ stage motions were measured for each of three combinations of actuator inputs. The calibration matrix was measured to be

$$
\left[\begin{array}{c}
\Delta x \\
\Delta y \\
\Delta \theta_{z}
\end{array}\right]=\left[\begin{array}{ccc}
9.8005 & -4.2330 & -6.5472 \\
0.3061 & -6.8919 & 8.5796 \\
0.0075 & 0.0053 & 0.0076
\end{array}\right]\left[\begin{array}{c}
i_{1} \\
i_{2} \\
i_{3}
\end{array}\right] .
$$

The input current has units of Amps, displacement has units of $\mu \mathrm{m}$, and rotation has units of radians. Figure 10 shows the measured quasistatic response of the nanopositioner in the X-and $\mathrm{Y}$-directions to calibrated commands in the X-direction.

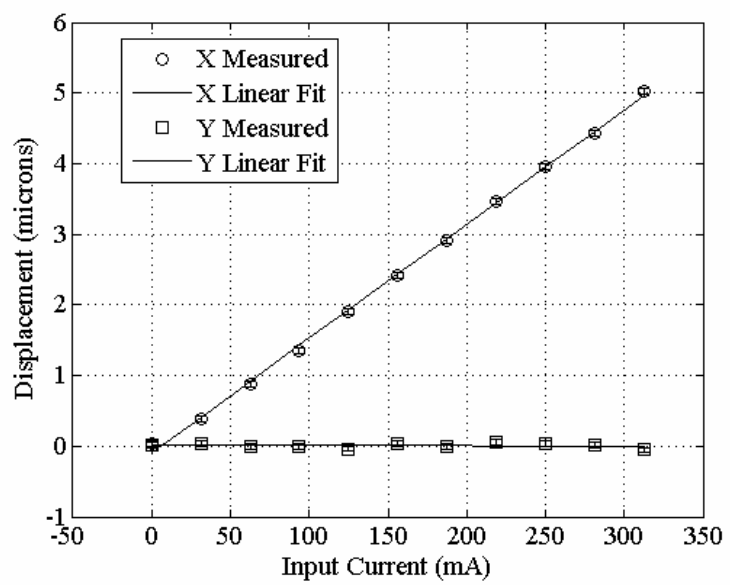

Figure 10: Measured in-plane quasistatic response to calibrated commands in the $X$-direction.

The measurement error bars of $\pm 40 \mathrm{~nm}$ are plotted in the figure. The input current plotted in the figure represents the maximum current applied to any of the three actuators. The data indicates that the nanopositioner has a unidirectional range-ofmotion that is larger than $5 \mu \mathrm{m}$ and therefore has a bidirectional range greater than $10 \mu \mathrm{m}$. The input current was limited to less than $350 \mathrm{~mA}$ in these experiments, while the temperature-limited 
maximum current is larger than $450 \mathrm{~mA}$. The nanopositioner may therefore have a useful range that is greater than $14 \mu \mathrm{m}$, but this has yet to be measured. Less than $100 \mathrm{~nm}$ of parasitic translation was recorded during the commanded translations.

The data in this section demonstrates that the parasitic motions that are due to parasitic moments and forces may be minimized during open-loop operation by linear calibration. In addition to the translational motion, the system was measured to have a calibrated angular range of more than 0.5 degrees in the $\theta_{z}$ direction.

\section{Out-of-Plane Step Response}

Out-of-plane motion in the Z-direction was measured prior to calibration with a laser vibrometer. Square wave inputs were commanded to one Z-actuator. The input square wave oscillated between 0 and $50 \mathrm{~mA}$, which is well below the actuator coil current limit of $450 \mathrm{~mA}$. Figure 11 shows the pre-calibrated, outof-plane step response.

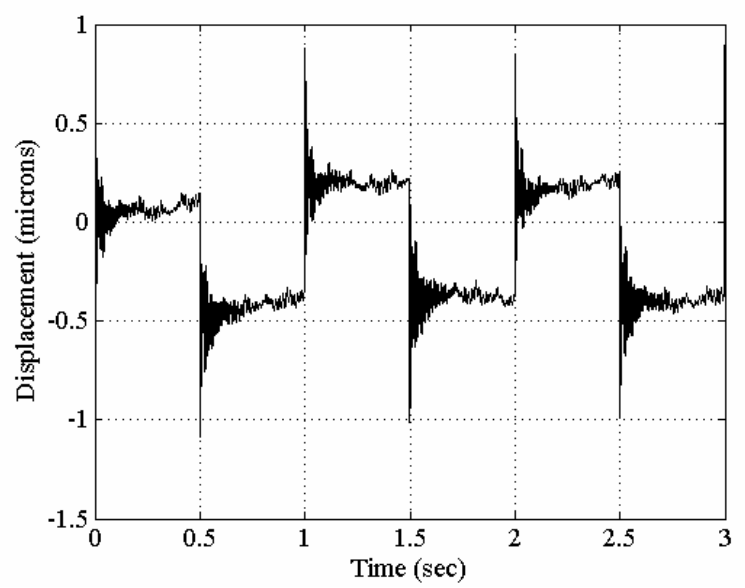

Figure 11: Measured out-of-plane response to pre-calibrated step commands in the out-of-plane Z-direction.

The data indicates that the nanopositioner has a unidirectional range of $0.5 \mu \mathrm{m}$ in the Z-direction with a calibration constant of 10 $\mu \mathrm{m} / \mathrm{A}$. The bidirectional range is therefore at least $1 \mu \mathrm{m}$. An input of $\pm 100 \mathrm{~mA}$ would result in $\pm 1 \mu \mathrm{m}$ of motion in the Z-direction.

The data in Figure 11 also indicates that system settling time is less than $250 \mathrm{~ms}$. The excessive settling time and greater than $200 \mathrm{~nm}$ drift may be due to thermally-induced error motions from power dissipated in the actuator coils. The power dissipation induces thermal bimorph bending of the flexure beams which may cause the observed drift. Experiments are in progress to investigate the effects of thermally-induced error motions that are caused by power dissipation within the microcoils.

\section{CONCLUSIONS}

This paper presented the design, microfabrication, and initial experimental characterization of a six-axis, meso-scale nanopositioner. The nanopositioner uses a silicon flexure bearing and a set of two-axis linear moving-coil actuators to enable inplane and out-of-plane motion in open-loop. The system was measured to have a range of motion of $10 \mu \mathrm{m}$ in the lateral directions, a range of $1 \mu \mathrm{m}$ in the out-of-plane direction, an angular range of 0.5 degrees, and a first mode resonant frequency at 900 Hz. Experiments are in progress to calibrate the system in six-axis and investigate the thermally-induced errors motions. The system is envisioned to provide a platform for future low-cost, highperformance instruments and equipment that are used in nano-scale research and nano-manufacturing.

\section{ACKNOWLEDGEMENTS}

This material is based upon work supported by the National Science Foundation under Grant 0348242 - PECASE: Research and Education Plans for Modeling and Design of Fixtures and SixAxis Manipulators for Nanomanufacturing. The authors would like to thank the staff of the MIT MTL for their guidance and use of microfabrication facilities, Nexx Systems for the use of their electroplating services, Professors Jeff Lang and Dennis Freeman for use of experimental equipment, and Polytec for the use of the laser vibrometer.

\section{REFERENCES}

[1] E. Eleftheriou, T. Antonakopoulos, G. K. Binnig, G. Cherubini, M. Despont, A. Dholakia, U. Durig, M. A. Lantz, H. Pozidis, H. E. Rothuizen, and P. Vettiger, "Millipede - a MEMS-based scanning-probe data-storage system," IEEE Transactions on Magnetics, 39, 2 (2003).

[2] A.P. Malshe, K. Virwani, K. P. Rajurkar, and D. Deshpande, "Investigation of nanoscale electro machining (nano-EM) in dielectric oil," presented at 55th CIRP General Assembly, Antalya, Turkey, 8/21-27/05.

[3] J.J. Choi, H. Park, K. Y. Kim, and J. U. Jeon, "Electromagnetic micro $\mathrm{x}-\mathrm{y}$ stage for probe-based data storage," Journal of Semiconductor Technology and Science, $1,1(2001)$.

[4] H. Rothuizen, U. Drechsler, G. Genolet, W. Haberle, M. Lutwyche, R. Stutz, R. Widmer, and P. Vettiger, "Fabrication of a micromachined magnetic $\mathrm{X} / \mathrm{Y} / \mathrm{Z}$ scanner for parallel scanning probe applications," Microelectronic Engineering, 53, 1 (2000).

[5] H. Rothuizen, M. Despont, U. Drechsler, G. Genolet, W. Haberle, M. Lutwyche, R. Stutz, and P. Vettiger, "Compact copper/epoxy-based electromagnetic scanner for scanning probe applications," Proceeding of the 2002 IEEE International Conference on MEMS, Las Vegas, NV, 1/2024/2002, IEEE 2002, pp. 582-585.

[6] M. L. Culpepper and S. Chen, "Design of a Compliant, Micro-scale, Six-axis Nanopositioner-The Micro-HexFlex," Precision Engineering, 30 (3) 314-24, 2006.

[7] M. L. Culpepper and G. Anderson, "Design of a Low-cost Nano-manipulator Which Utilizes a Monolithic, Spatial Compliant Mechanism," Precision Engineering, 28 (4), 46982, 2004.

[8] D. Golda and M. L. Culpepper, "Two-axis electromagnetic moving-coil micro-actuator," Proceedings of the 2006 ASME International Mechanical Engineering Conference and Exposition, Chicago, IL, 11/5-10/2006, ASME 2006.

[9] D. S. Golda "Design of High-speed Meso-scale Nanopositioners Driven by Electromagnetic Actuators," Ph.D. Thesis, Massachusetts Institute of Technology, February 2008. 